\title{
Equatorial electron cyclotron port plug neutronic analyses for the EU DEMO
}

\author{
Aljaž Čufar ${ }^{\mathrm{a}}$, Alessandro Bruschi ${ }^{\mathrm{b}}$, Ulrich Fischer ${ }^{\mathrm{c}}$, Thomas Franke ${ }^{\mathrm{d}, \mathrm{e}}$, Gustavo Granucci $^{\mathrm{b}}$, \\ Giovanni Grossetti ${ }^{\mathrm{c}}$, Ivan A. Kodeli ${ }^{\mathrm{a}}$, Denise Trombetta ${ }^{\mathrm{f}}$, Rosaria Villari ${ }^{\mathrm{g}}$ \\ ${ }^{a}$ Reactor Physics Department, Jožef Stefan Institute, Jamova cesta 39, SI-1000, Ljubljana, Slovenia \\ ${ }^{b}$ Istituto di Fisica del Plasma "P. Caldirola", CNR, Milano, Italy \\ ${ }^{c}$ Karlsruhe Institute of Technology, D-76344 Eggenstein-Leopoldshafen, Karlsruhe, Germany \\ ${ }^{d}$ Max-Planck-Institut für Plasmaphysik, Boltzmannstr. 2, D-85748 Garching, Garching, Germany \\ ${ }^{e}$ EUROfusion Programme Management Unit, PPPT, Boltzmannstr. 2, D-85748 Garching, Germany \\ ${ }^{f}$ University LA Sapienza of Rome, DIAEE department, I-00186 Rome, Italy \\ ${ }^{g}$ ENEA, Department of Fusion and Nuclear Safety Technology, I-00044 Frascati (Rome), Italy
}

\begin{abstract}
Within the Power Plant Physics and Technology (PPPT) programme in the EUROfusion Consortium design activities are currently in progress for the development of a DEMOnstration Fusion Power Plant (DEMO). The design of the machine and the integration of in-vessel components require neutronics analyses to verify the tritium selfsufficiency, the shielding requirements, and the structural integrity of its components. In particular, the effect of penetrations in the blanket and in equatorial port plug introduced by the electron cyclotron (EC) heating system, namely due to openings for the antenna waveguides (WGs), were analyzed. In this study three-dimensional MCNP calculations were conducted for the pre-conceptual designs of the EC port plugs and shielding optimization were performed in order to ensure that the DEMO design limits are not exceeded.

Two configurations of the EC heating system were performed using a DEMO Water Cooled Lithium Lead (WCLL) with integrated EC configurations model of a half of the sector (i.e. $10^{\circ}$ model) and relevant ones repeated with a full sector model (i.e. $20^{\circ}$ model) to test the reliability of results. Additionally, the effect of the radiation on the WGs closest to the plasma was analyzed as well as the impact on Tritium Breeding Ratio (TBR).
\end{abstract}

Keywords: DEMO, equatorial port plug, electron cyclotron, nuclear heating, material damage

\section{Introduction}

In support of the integration of EC heating system [1] neutronic simulations were performed to check the design requirements are met and to trigger potential design optimizations. In this process the reduction in the $\mathrm{T}$ breeding performance of the breeding blanket as well as shielding performance of the design was assessed.

Two different remote steering EC system designs were analyzed both consisting of 8 waveguides (WGs) [2]. Due to penetrations in the tritium breeding blanket (TBB) and port plug it was expected that further shielding optimization would be required to keep the nuclear heating of the toroidal field (TF) coils and the neutron induced damage of the exposed part of the vacuum vessel (VV) below their design limits. Additionally, the nuclear heating and the neutron damage to the exposed parts of the EC WGs were assessed.

To reduce the CPU time requirements, first a halfsector model $\left(10^{\circ}\right)$ was used while later the important results were confirmed using a more suitable full-sector model $\left(20^{\circ}\right)$. In the present paper the half-sector model was used only for assessment of TF coil heating which represents a conservative assessment. The values for the TBR and VV damage are from a full-sector model simulations.

\section{Configurations and models}

\subsection{EC configurations}

The first analyzed EC configuration consisted of two rows of four WGs (EC\#1, Figure 1) and the second of a single column of eight WGs (EC\#2, Figure 2). The inner dimensions of the rectangular copper WGs were in both cases $63.5 \mathrm{~mm} \times 63.5 \mathrm{~mm}$ and the thickness of their walls $2 \mathrm{~cm}$.

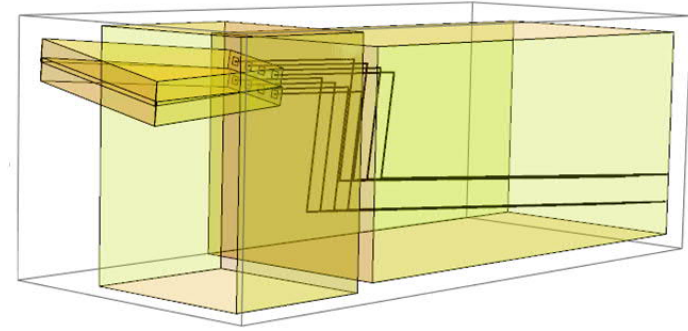

Figure 1. EC\#1 waveguide configuration $(2 \times 4 \mathrm{WGs})$. 


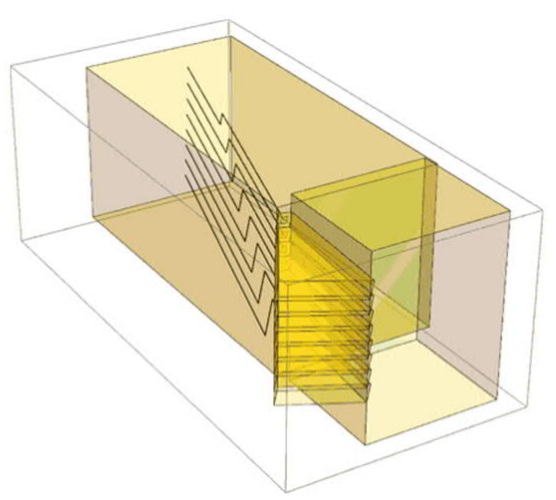

Figure 2. EC\#2 waveguide configuration ( $8 \times 1 \mathrm{WGs})$.

\subsection{DEMO models}

The DEMO model used in the neutronic analyses was based on the generic model [3][4] with modifications needed for the equatorial port plug containing the EC heating system (Figure 3). For both configurations the part of the tritium breeding blanket in front of the equatorial port was modified to include penetration for EC WGs. McCad [5] and SuperMC [6] CAD to MCNP [7] conversion tools were used for model preparation and ADVANTG [8] was used to speed up the simulations.

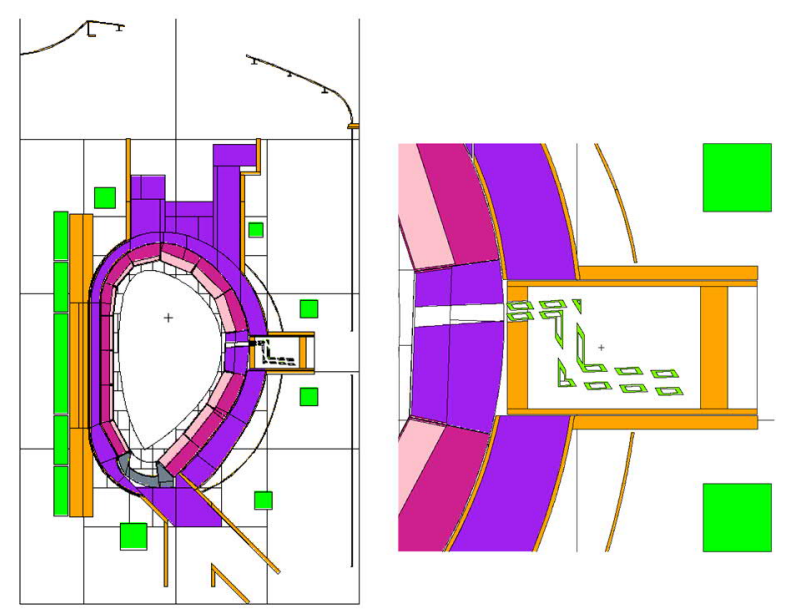

Figure 3. Vertical cross-section of the MCNP model of DEMO used in analyses (left) and a closer view of the initial EC\#1 port plug configuration (right).

\subsection{Relevant design limits}

The design limits relevant for this work are presented in Table 1 and the lifetime of DEMO is considered as 6 full power years (FPY) of operation.

Table 1. Design limits for the assessed factors [9][10].

\begin{tabular}{lc}
\hline Parameter & Value \\
\hline Tritium breeding ratio (TBR) & $\geq 1.10$ \\
Peak nuclear heating & $<50 \mathrm{~W} / \mathrm{m}^{3}$ \\
of TF coils (NH TFC) & \\
Peak displacement damage & $<2.75 \mathrm{dpa} /$ lifetime \\
in vacuum vessel (VV dpa) & \\
\hline
\end{tabular}

The effect on the TBR for the single module segment WCLL blanket concept [11] was assessed. The value for the reactor without EC port plug (baseline) was 1.156 and the values for both EC configurations are presented in Table 2 for a case with $5 \mathrm{EC}$ systems providing up to $50 \mathrm{MW}$ of plasma heating during operation [12][13]. This assessment used full sector $\left(20^{\circ}\right)$ model and took into account that only 5 out of 18 sectors include the system. Analysis also included an estimation of the effect on the $\mathrm{T}$ breeding performance in the sectors next to the EC system. The tritium breeding performance of the sectors next to sectors with EC port plug were assessed as a mean value between the EC and the baseline while the sectors not neighboring on the EC sectors were taken as unaffected (baseline values) [12]. The resulting TBR value for EC\#1 is just below the acceptable 1.1 while the EC\#2 is sufficiently high to allow some margin needed for integration of other systems. The reason for the difference in TBR performance is that in the case of EC\#1 the whole part of the TBB in front of the port plug was replaced with a shield while the position of WGs in EC\#2 configuration close to the edge of the blanket module allowed for significantly smaller displacement of the breeding material with shield. These two configurations represent two extreme cases where the TBB volume in front of the port is either completely lost (EC\#1) or almost completely usable (EC\#2) for tritium breeding.

Table 2. First results with initial design of the EC port plugs.

\begin{tabular}{lcc}
\hline Parameter & Value EC\#1 & Value EC\#2 \\
\hline TBR & 1.099 & 1.126 \\
NH TFC $\left[\mathrm{W} / \mathrm{m}^{3}\right]$ & 400 & 900 \\
VV dpa $[\mathrm{dpa} /$ lifetime $]$ & 0.5 & 10 \\
\hline
\end{tabular}

At their narrowest part the cut-outs in the TBB and port plug for EC\#1 and EC\#2 are approx. $67 \mathrm{~cm} \times 32 \mathrm{~cm}$ and $26 \mathrm{~cm} \times 122 \mathrm{~cm}$ respectively. Due to these large openings the shielding performance was found insufficient for both cases. The peak values of TF coil heating (Table 2) were thus 8-times (Figure 4) and 18times too high for EC\#1 and EC\#2 respectively. Additionally, it was found that while the peak values of the neutron damage in VV increased for both cases (Table 2 ), the position of the opening in the blanket for EC\#2 close to the edge of the port especially increased the neutron damage in the exposed part of the VV above the limit of $2.75 \mathrm{dpa} /$ lifetime. On the other hand, the peak values for EC\#1 were found to be well below this limit (Figure 5) as the distance between the opening and the VV means that the peak values in the VV are $0.09 \mathrm{dpa} / \mathrm{FPY}$ or $0.5 \mathrm{dpa} /$ lifetime, well within the design limits. Both Figure 4 and Figure 5 show profiles peaked in the vicinity of the opening for the EC WGs.

\subsection{First results}




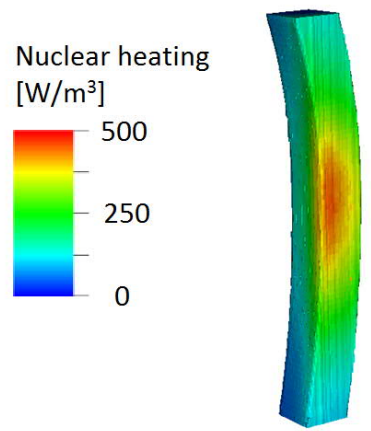

Figure 4. An initial determination of the nuclear heating in TF coils for EC\#1.

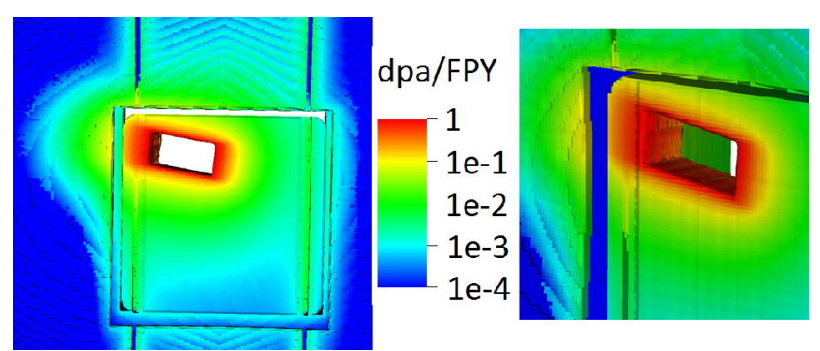

Figure 5. Neutron displacement damage in the stainless steel of the VV and port plug for the EC\#1.

The plasma facing part of the WGs is exposed to neutrons and neutron induced gamma radiation. To assess this the neutron damage and nuclear heating in the $\mathrm{Cu}$ WGs were assessed. Results are presented in Table 3 and the values will be used as a basis for the selection of suitable WG materials and their lifetimes.

Table 3. Peak values of neutron induced displacement damage and nuclear heating in plasma-facing part of the $\mathrm{Cu}$ waveguides.

\begin{tabular}{lcc}
\hline Parameter & EC case & Peak value \\
\hline Neutron damage & EC\#1 & 1.5/FPY or 9.1/lifetime \\
[dpa] & EC\#2 & $3.6 /$ FPY or 21.6/lifetime \\
Nuclear heating & EC\#1 & 2.3 \\
{$\left[\mathrm{MW} / \mathrm{m}^{3}\right]$} & EC\#2 & 4.8 \\
\hline
\end{tabular}

Additionally, nuclear heating maps for the port plug region were assessed (Figure 6). The effect of the large opening in the blanket is clearly visible as elevated heat loads extend all the way to the port plug. The values are within the technical limits.

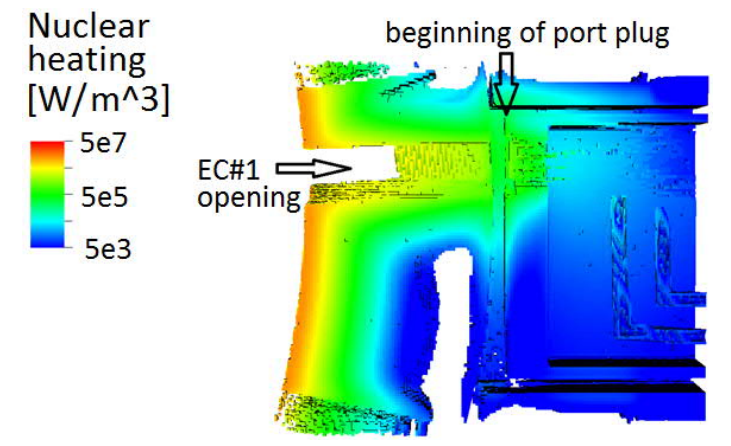

Figure 6. Nuclear heating maps for EC\#1 configuration. Maximum heating loads of $1.3 \times 10^{7}$ and $1 \times 10^{6} \mathrm{~W} / \mathrm{m}^{3}$ were found for the first wall and front part of the port plug respectively. Values in the opening are between these two values.

\section{Shielding optimization}

The shielding performance of both EC configurations is insufficient and additional shielding is required. This was expected due to the size of the penetrations and the amount of shielding material displaced by the EC systems and their port plug. Different shielding strategies were tested and possible solutions found. In these analyses, additional shielding blocks (60\% stainless steel, $40 \%$ water) were positioned in different locations inside the port plug, the effect of the increased port walls tested, and the size of the gap between the port plug and the port varied.

\subsection{Nuclear heating in TF coils}

Adding sufficient amount of shielding to the port plug can reduce the peak values of nuclear heating in TF coils below the design limits. However, putting a lot of material into the port plug increases its weight and can make such design impractical. Because of that, different shielding strategies were considered for EC\#1 to find different possible design configurations:

- $40 \mathrm{~cm}$ shield in front part of the port plug (Figure 7, left) and $20 \mathrm{~cm}$ shield right after the dogleg (Figure 7, right).

- Doubling the thickness of the port wall (DPW configuration) from $20 \mathrm{~cm}$ to $40 \mathrm{~cm}$ in order to reduce the peak nuclear heating of TF coils without adding weight to the port plug.

- Combination of thicker port wall and $20 \mathrm{~cm}$ shield after WG dogleg

- Reduction in the size of the opening in the port plug around the WGs (Figure 8).

Results of these shielding cases for EC\#1 are presented in Table 4. While multiple strategies for decreasing of the nuclear heating in the TF coils were identified, a combination of multiple strategies (e.g. shield + thicker port wall) is likely more suitable than simply adding a large amount of shielding material to the port plug. However, other analyses such as shut down dose rate (SDDR) analyses in relevant regions are needed to further confirm the suitability of the design choices. For example while the size of the gap between the port plug and the port wall was found to have minimal effect on the peak TF coil heating it could potentially significantly increase the SDDR behind the port plug. After all, in the final design all the design requirements must be met. 


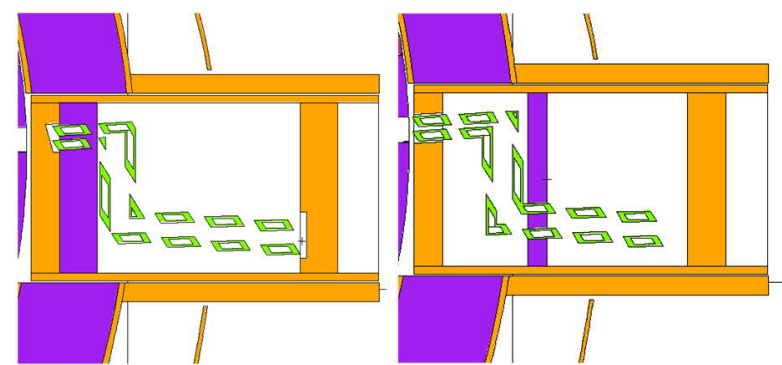

Figure 7. Two shield options in the port plug. $40 \mathrm{~cm}$ shield before the WG dogleg (40 cm shield, left) and $20 \mathrm{~cm}$ shield after dogleg (20 cm shield after dogleg (DL), right).

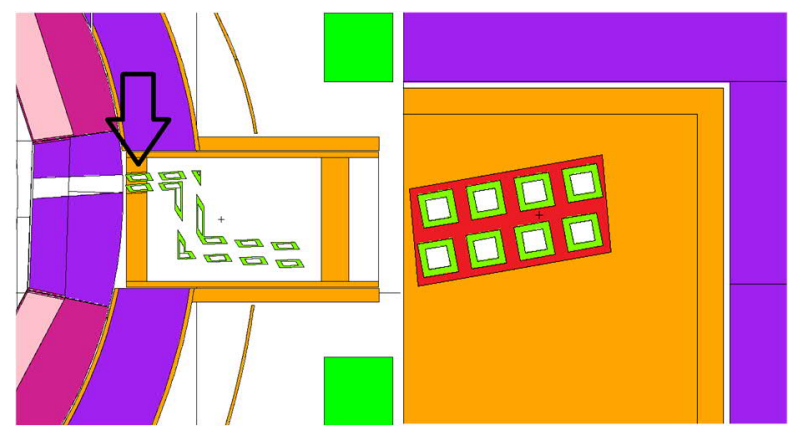

Figure 8. The location of the opening around WGs (left) and a (red) cell used to close it up (right). (Color online)

Additionally some analyses were performed for EC\#2. It was found that a $70 \mathrm{~cm}$ shield in the front part of the port plug results in a nuclear heating of $11 \mathrm{~W} / \mathrm{m}^{3}$ in the TF coil - similar levels to those obtained for a $40 \mathrm{~cm}$ shield in EC\#1. Other shielding strategies, e.g. thicker port wall and reduced opening for WGs, are expected to produce similar relative reductions to the ones from EC\#1.

Table 4. Peak values of nuclear heating in TF coils for EC\#1 and different shielding strategies.

\begin{tabular}{cc}
\hline Shielding option & NH TFC $\left[\mathrm{W} / \mathrm{m}^{3}\right]$ \\
\hline Original & 400 \\
$40 \mathrm{~cm}$ front shield & 12 \\
$20 \mathrm{~cm}$ shield after dogleg & 70 \\
Double port wall (DPW) & 130 \\
No opening around WGs & 170 \\
DPW $+20 \mathrm{~cm}$ shield after DL & 20 \\
\hline
\end{tabular}

\subsection{Neutron damage in vacuum vessel (EC\#2)}

Different approaches were considered in order to reduce the neutron damage to the vacuum vessel due to the opening for the EC\#2 configuration (Figure 9).

While adding some shield might be possible it would be difficult to sufficiently reduce the damage due to geometrical requirements of the opening - the opening around WGs is based on the operating requirements of the EC system. An alternative solution would be to exclude the exposed area from the VV. This might be achieved through increasing the size of the opening for the port beyond the region exposed to excessive neutron radiation and then reducing the opening by stainless steel attachment which is not part of the VV. In this way, the requirements for the exposed part of the stainless steel is less strict than for the $\mathrm{VV}$ which is a crucial lifetime component. It was found that excluding $25 \mathrm{~cm}$ of the VV edge reduces the peak value below the limit (exclusion zone is shown as white square in Figure 9).

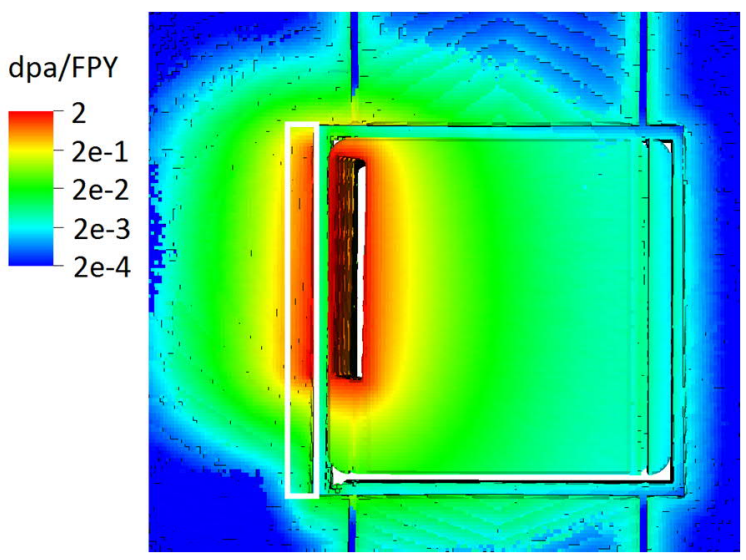

Figure 9. Map of neutron damage in stainless steel parts of the EC\#2 port plug and VV.

\section{Conclusion}

Neutronic analyses were performed in support of the development and integration of the equatorial port plug for the remote steering EC heating system. Various design parameters were analyzed and optimizations of the shielding configuration were suggested. The analyzed parameters were the TBR, nuclear heating in TF coils, and neutron damage in VV. These analyses gave insight into the problem, identified some potentially suitable shielding solutions for the analyzed cases, and showed some shielding strategies useful for future system designs. It is important to understand that while simply filling up large parts of the port plug is a valid shielding strategy it can result in excessive weight of the port plug and alternative strategies have to be considered. The reduction in the size of the opening around EC waveguides and the increase in the thickness of the port wall are two examples of such alternative options. To meet all of the design requirements in the final design it is likely that multiple shielding strategies will have to be implemented at the same time.

\section{Acknowledgments}

This work has been carried out within the framework of the EUROfusion Consortium and has received funding from the Euratom research and training programme 20142018 under grant agreement No. 633053. The views and opinions expressed herein do not necessarily reflect those of the European Commission.

\section{References}

[1] T. Franke et al., Initial port integration concept for EC and NB systems in EU DEMO tokamak, contribution at SOFT-2018.

[2] S. Garavaglia et al., EU DEMO EC sysem preliminary conceptual design, In press, FED, 2018.

[3] P. Pereslavtsev, Generic neutronic analyses for DEMO including the development of a global MCNP model, final report, EFDA_D_2M7HN3, (2016). 
[4] U. Fischer et al., Methodological approach for DEMO neutronics in the European PPPT programme: Tools, data and analyses, FED 123, pp. 26-31, (2017).

[5] L. Lu et al., Improved algorithms and advanced features of the CAD to MC conversion tool McCad, Fusion Engineering and Design 89, pp. 1885-1888, (2014).

[6] Y. Wu, FDS Team, CAD-based interface programs for fusion neutron transport simulation, Fusion Engineering and Design 84 (2009) 1987-1992.

[7] F. Brown et al, Verification of MCNP5-1.60, LA-UR-1005611, Los Alamos National Laboratory (2010).

[8] S.W. Mosher et al., ADVANTG - An Automated Variance Reduction Parameter Generator, ORNL/TM-2013/416 Rev. 1, Oak Ridge National Laboratory, 2015.

[9] U. Fischer et al, Neutronics requirements for a DEMO fusion power plant, Fusion Engineering and Design 98-99 (2015), pp. 2134-2137.

[10] P. Pereslavtsev and R. Villari, DEMO Nuclear Analysis Handbook (NAH), EUROfusion report, 2016, EFDA_D_2MQ22W.

[11] R. Villari, G. Mariano, WCLL neutronics, ENEA \& EUROfusion technical report 30032016.

[12] D. Trombetta, Neutronic Analyses with MCNP Code for the Design and Integration of Electron Cyclotron Heating System in Demo Fusion Reactor, Master thesis, Sapienza University of Rome, 2017.

[13] A. Čufar, Final Report on Deliverable: Neutronics study 2017 of EC equatorial port plug solutions for DEMO1, EFDA_D_2MP8VW, EUROfusion (2018). 